\title{
BOUNDED PROJECTIVE FUNCTIONS AND HYPERBOLIC METRICS WITH ISOLATED SINGULARITIES
}

\author{
Bo Li, Long Li and Bin $\mathrm{Xu}$ \\ Chinese Academy of Science, USTC, Wu Wen-Tsun Key Laboratory of Mathematics \\ and University of Science and Technology of China, School of Mathematical Sciences \\ No. 96 Jinzhai Road, Hefei, Anhui Province 230026, P. R. China; ilozyb@mail.ustc.edu.cn \\ University of Iceland, Science Institute \\ Dunhaga 5, 107 Reykjavík, Iceland; longli@hi.is \\ Chinese Academy of Science, USTC, Wu Wen-Tsun Key Laboratory of Mathematics \\ and University of Science and Technology of China, School of Mathematical Sciences \\ No. 96 Jinzhai Road, Hefei, Anhui Province 230026, P. R. China; bxu@ustc.edu.cn
}

\begin{abstract}
We establish a correspondence on a Riemann surface between hyperbolic metrics with isolated singularities and bounded projective functions whose Schwarzian derivatives have at most double poles and whose monodromies lie in $\operatorname{PSU}(1,1)$. As an application, we construct explicitly a new class of hyperbolic metrics with countably many singularities on the unit disc.
\end{abstract}

\section{Introduction}

In this manuscript we call a conformal metric of Gaussian curvature -1 on a Riemann surface a hyperbolic metric. Hyperbolic metrics with isolated singularities are the main objects of our investigation. Such kind of problems have root in the classical Uniformization Theory [7, Chapter IV] and the history of them could go back to Picard [22]. Based on the celebrated result by Nitsche [21] and Heins [11] that an isolated singularity is either a cone singularity or a cusp one, on a Riemann surface, not necessarily compact, we establish a correspondence in Theorem 2.2 between hyperbolic metrics with isolated singularities and bounded projective functions whose Schwarzian derivatives have at most double poles and whose monodromies lie in $\operatorname{PSU}(1,1)$. Hence it reduces differential geometric problems about hyperbolic metrics into the corresponding complex analytical ones, and particularly makes sense on noncompact Riemann surfaces since people have already known the classical existence theorem (Theorem 2.1) of hyperbolic metrics with finitely many singularities on compact Riemann surfaces by Heins [11, Chapter II]. The referee kindly pointed out to us that actually this correspondence is a corollary of a more general result [18, Theorem 1.2], where Kraus and Roth investigated conformal metrics of negative Gaussian curvature extending continuously to the isolated singularities. However, our proof of the correspondence is much shorter and substantially different from that of [18, Theorem 1.2]. Moreover, by using the correspondence, we construct in Example 2.1 a new class of hyperbolic metrics with infinitely many singularities on the disc. We are also informed friendly by the referee that hyperbolic metrics with cone angles of $2 \pi$ times positive integers on the disc are relevant to a major problem [17] in the theory of Bergman spaces, which has been open for 50 years or so.

https://doi.org/10.5186/aasfm.2020.4539

2010 Mathematics Subject Classification: Primary 51M10; Secondary 35J61, 34M35.

Key words: Hyperbolic metric with singularities, projective function. 
We prepare the notions, including the differential geometric concept of cone singularities and cusp one, provide the details of the background and give the complete statements for Theorem 2.1, Theorem 2.2 and Example 2.1 in the next section. In Section 3, we give the complex analytical definition (Definition 3.1) of both cone singularities and cusp one of a hyperbolic metric and prove that the differential geometric definition implies the complex analytical one by the PDE method. As a consequence, we obtain the necessary part of Theorem 2.2. In the last section, we prove that complex analytical definition implies the differential geometric one, which also implies the sufficient part of Theorem 2.2. We also provide in this section the detail of Example 2.1 and propose three questions.

\section{Background and the main results}

Let $X$ be a compact Riemann surface and $D=\sum_{j=1}^{n}\left(\theta_{j}-1\right) P_{j}$ be an $\mathbf{R}$-divisor on $X$ such that $1 \neq \theta_{j} \geq 0$ and $P_{1}, \cdots, P_{n}$ are $n$ distinct points on $X$. We call $d s^{2}$ a conformal metric representing $D$ on $X$ if $d s^{2}$ is a smooth conformal metric on $X \backslash \operatorname{supp} D:=X \backslash\left\{P_{1}, \cdots, P_{n}\right\}$ and in a neighborhood $U_{j}$ of $P_{j}$,ds $s^{2}$ has form $e^{2 u_{j}}|d z|^{2}$, where $z$ is a local complex coordinate defined in $U_{j}$ centered at $P_{j}$ and the smooth real valued function

$$
v_{j}:= \begin{cases}u_{j}-\left(\theta_{j}-1\right) \ln |z| & \text { if } \theta_{j}>0 \\ u_{j}+\ln |z|+\ln (-\ln |z|) & \text { if } \theta_{j}=0\end{cases}
$$

on $U_{j} \backslash\left\{P_{j}\right\}$ extends to a continuous function on $U_{j}$. We also say that $d s^{2}$ has a cone singularity of angle $2 \pi \theta_{j}$ at $P_{j}$ when $\theta_{j}>0$, and it has a cusp singularity at $P_{j}$ when $\theta_{j}=0$. We may think of the above definition of cone/cusp singularity a differential geometric one. We call $d s^{2}$ a hyperbolic metric representing $D$ on $X$ if $d s^{2}$ is a conformal metric representing $D$ on $X$ whose Gaussian curvature equals -1 identically on $X \backslash \operatorname{supp} D$. There also exists a unified complex analytical definition for both cone singularities and cusp one of a hyperbolic metric (Definition 3.1). We prove in Lemma 3.1 that these two definitions coincide with each other when we are considering hyperbolic metrics. Nitsche [21] and Heins [11, § 18] proved that an isolated singularity of a hyperbolic metric must be either a cone singularity or a cusp one. By the Gauss-Bonnet formula, if $d s^{2}$ is a hyperbolic metric representing $D=\sum_{j=1}^{n}\left(\theta_{j}-1\right) P_{j}$ on $X$, then there holds

$$
\chi(X)+\sum_{j=1}^{n}\left(\theta_{j}-1\right)<0,
$$

where $\chi(X)$ is the Euler number of $X$. Heins [11] studied the properties of S-K metrics and applied it to showing

Theorem 2.1. [11, Chapter II] There exists a unique hyperbolic metric representing an $\mathbf{R}$-divisor $D=\sum_{j=1}^{n}\left(\theta_{j}-1\right) P_{j}$ with $\theta_{j} \geq 0$ on a compact Riemann surface $X$ if and only if $\chi(X)+\sum_{j=1}^{n}\left(\theta_{j}-1\right)<0$.

Actually the history of this problem goes back to Picard [22], who studied the hyperbolic metrics with cone singularities. After Heins' work [11], both McOwen [20] and Troyanov [26], who were unaware of Heins' work [11] apparently, proved the theorem for the case of $\theta_{j}>0$ by using different PDE methods. Moreover, the hyperbolic metrics on the Riemann sphere with three singularities were expressed 
explicitly in $[1,19,28]$ by using the Gauss hypergeometric functions and some refined properties of the metrics were also studied there.

In this manuscript, by using developing map [24, §3.4] and [4, Sections 2-3] and Complex Analysis, we investigate more general hyperbolic metrics with isolated singularities on Riemann surfaces, which are not necessarily compact. To this end, we need to prepare some notions at first. Let $\mathfrak{X}$ be a Riemann surface and $\mathfrak{D}=$ $\sum_{j}\left(\theta_{j}-1\right) \mathfrak{P}_{j}$ an $\mathbf{R}$-divisor on $\mathfrak{X}$ such that $\theta_{j} \geq 0$ and $\mathfrak{P}_{1}, \mathfrak{P}_{2}, \cdots$ are mutually distinct points on $\mathfrak{X}$, which form a closed and discrete subset of $\mathfrak{X}$. We denote $\left\{\mathfrak{P}_{1}, \mathfrak{P}_{2}, \cdots\right\}$ by supp $\mathfrak{D}$, which is at most countable. We could give the definition of the conformal metric representing $\mathfrak{D}$ on $\mathfrak{X}$ in the similar way as the first paragraph of this section. We say a multi-valued locally univalent meromorphic function $f: S \rightarrow$ $\mathbf{P}^{1}:=\mathbf{C} \cup\{\infty\}$ on a Riemann surface $S$ a projective function if the monodromy of $f$ lies in the group $\operatorname{PSL}(2, \mathbf{C})$ consisting of all Möbius transformations. We say a projective function $f$ on $\mathfrak{X} \backslash \operatorname{supp} \mathfrak{D}$ compatible with $\mathfrak{D}$ if and only if the Schwarzian derivative $\{f, z\}=\left(\frac{f^{\prime \prime}(z)}{f^{\prime}(z)}\right)^{\prime}-\frac{1}{2}\left(\frac{f^{\prime \prime}(z)}{f^{\prime}(z)}\right)^{2}$ of $f$ has the form of $\frac{1-\theta_{j}^{2}}{2 z^{2}}+\frac{b_{j}}{z}+h_{j}(z)$ near each $\mathfrak{P}_{j}$, where $z$ is a complex coordinate centered at $\mathfrak{P}_{j}, b_{j}$ is a constant, and $h_{j}(z)$ is holomorphic near $\mathfrak{P}_{j}$. We note that both the constant $b_{j}$ and the holomorphic function $h_{j}(z)$ depend on the choice of the complex coordinate $z$, but the principal singular term $\frac{1-\theta_{j}^{2}}{2 z^{2}}$ of $\{f, z\}$ does not.

Theorem 2.2. There exists a hyperbolic metric $d s^{2}$ representing an $\mathbf{R}$-divisor $\mathfrak{D}=\sum_{j}\left(\theta_{j}-1\right) \mathfrak{P}_{j}$ with $\theta_{j} \geq 0$ on a Riemann surface $\mathfrak{X}$ if and only if there exists a projective function $f: \mathfrak{X} \backslash \operatorname{supp} \mathfrak{D} \rightarrow \mathbf{D}:=\{w \in \mathbf{C}:|w|<1\}$ such that $f$ is compatible with $\mathfrak{D}$ and the monodromy of $f$ lies in the holomorphic automorphism group

$$
\operatorname{PSU}(1,1)=\left\{w \longmapsto \frac{a w+b}{\bar{b} w+\bar{a}}: a, b \in \mathbf{C},|a|^{2}-|b|^{2}=1\right\}
$$

of D. Moreover, $d s^{2}$ coincides with the pullback $f^{*} g_{\mathrm{st}}$ of the standard hyperbolic metric $g_{\mathrm{st}}:=\frac{4|d w|^{2}}{\left(1-|w|^{2}\right)^{2}}$ on $\mathbf{D}$ by $f$. We say $f$ a developing map of the metric $d s^{2}$, which is uniquely determined up to a post-composition with an automorphism of $\mathbf{D}$.

Remark 2.1. Luo [16] also mentioned the same result as Theorem 2.2 for hyperbolic metrics with only cone singularities on compact Riemann surfaces. Kraus-Roth [18, Theorem 1.2] proved a more general result than Theorem 2.2 where they investigated conformal metrics with negative Gaussian curvature extending continuously to the isolated singularities. Our proof of Theorem 2.2 is an alternative and much shorter one for the hyperbolic case of their theorem. In particular, ours is reduced to showing that the differential geometric definition coincides with the complex analytical one for cusp singularity of a hyperbolic metric. The proof of the coincidence is interesting because we need to do some subtle analysis for a second order nonlinear elliptic partial differential equation.

Remark 2.2. Chen, Wang, Wu and the last author [4, Theorem 3.4] proved an analogue of Theorem 2.2 for cone spherical (constant curvature one) metrics on compact Riemann surfaces, which motivated us to come up with Theorem 2.2. For the convenience of the readers, we state a more general version of [4, Theorem 3.4] as follows: There exists a conformal cone spherical metric $\widetilde{d s^{2}}$ representing an $\mathbf{R}$-divisor $\mathfrak{D}=\sum_{j}\left(\theta_{j}-1\right) \mathfrak{P}_{j}$ with $\theta_{j}>0$ on a Riemann surface $\mathfrak{X}$ if and only if there exists a projective function $\tilde{f}: \mathfrak{X} \backslash \operatorname{supp} \mathfrak{D} \rightarrow \mathbf{P}^{1}$ such that $\tilde{f}$ is compatible with $\mathfrak{D}$ and the 
monodromy of $\tilde{f}$ lies in the group

$$
\operatorname{PSU}(2)=\left\{w \longmapsto \frac{a w+b}{-\bar{b} w+\bar{a}}: a, b \in \mathbf{C},|a|^{2}+|b|^{2}=1\right\}
$$

consisting of all the Möbius transformations preserving the standard spherical metric $\frac{4|d w|^{2}}{\left(1+|w|^{2}\right)^{2}}$ on the Riemann sphere $\mathbf{P}^{1}$. Moreover, $\widetilde{d s^{2}}=\tilde{f}^{*}\left(\frac{4|d w|^{2}}{\left(1+|w|^{2}\right)^{2}}\right)$. Of course, the proof of the preceding statement in [4, Theorem 3.4] was much simpler than that of Theorem 2.2 because a spherical metric with finite area has no cusp singularity.

As an application of Theorem 2.2, we find the following new example of hyperbolic metrics with countably many singularities.

Example 2.1. Let $\sum_{j=1}^{\infty} a_{n}$ be a convergent series of positive numbers and $\left\{z_{j}\right\}_{j=1}^{\infty}$ a closed discrete subset of $\mathbf{D}$. Then $h(z):=\sum_{j=1}^{\infty} \frac{a_{j}}{z-z_{j}}$ is a meromorphic function on $\mathbf{D}$ and there exists a real number $\lambda_{0}$ and a one-parameter family $\left\{d s_{\lambda}^{2}: \lambda>\lambda_{0}\right\}$ of hyperbolic metrics representing the same $\mathbf{Z}$-divisor $\mathfrak{D}=(h)$ on $\mathbf{D}$. That is, these metrics have cusp singularities at $z_{j}$ 's and a cone singularity of angle $2 \pi\left(1+\operatorname{ord}_{w}(h)\right)$ at each zero $w$ of $h$. We obtain a similar statement if we use the finite sum $\sum_{j=1}^{N} a_{n}$ of positive numbers and a finite subset $\left\{z_{j}\right\}_{j=1}^{N}$ of $\mathbf{D}$, where $\sum_{j=1}^{N} \frac{a_{j}}{z-z_{j}}$ has $(N-1)$ zeros (counting multiplicities) on $\mathbf{D}$.

It is an easy observation that $h$ has no zero on $\{z \in \mathbf{C}:|z| \geq 1\}$. Borcea [3] proposed a conjecture that $h$ has infinitely many zeros in $\{z \in \mathbf{C}:|z| \geq 1\}$ if $z_{k}$ converges to a point on the unit circle $\{z \in \mathbf{C}:|z|=1\}$, which was disproved by Langley [13]. Langley op. cit. also gave a sufficient condition about $\left\{z_{j}\right\}$ under which $h(z)$ has infinitely many zeros converging to a point on the unit circle $\{z \in \mathbf{C}:|z|=$ $1\}$. There also exist a few of recent papers $[5,6,14,15]$ concerned zeros of $h(z)$. We show for

$$
h_{0}(z)=\sum_{j=1}^{\infty} \frac{1}{2 j^{3}(2 j+1)} \cdot \frac{1}{z-\left(1-\frac{1}{2 j-1}\right)}
$$

that there exists a unique zero $w_{j}$ of $h_{0}(z)$ lying on the interval $\left(1-\frac{1}{2 j-1}, 1-\frac{1}{2 j+1}\right)$ for all $j=1,2, \cdots$ and $\left\{w_{k}\right\}$ forms the zero set of $h_{0}(z)$.

Remark 2.3. Using the punctured disc case of Theorem 2.2, Feng, Shi and the last author [8] obtained an explicit local model of an isolated singularity of a hyperbolic metric in a suitably chosen complex coordinate around the singularity. Also motivated by Theorem 2.2, Feng, Shi and the last author [9] found the preceding local model by merely using Complex Analysis. Moreover, based on the correspondence in Theorem 2.2 and following the ideas in [4, 23], Feng, Shi, Song and the last author [10] have been investigating systematically new hyperbolic metrics with isolated singularities on noncompact Riemann surfaces by using both subharmonic functions and meromorphic (quadratic) differentials.

\section{The complex analytical definition}

At first we give the complex analytical definition of cone/cusp singularity of a hyperbolic metric. Consider a hyperbolic metric $d s^{2}=e^{2 u}|d z|^{2}$ on the punctured disc $U^{*}:=U-\{0\}=\{0<|z|<1 / 2\}$, where $U=\{|z|<1 / 2\}$. By the similar argument as [4, Lemma 2.1], there exists a projective function $f: U^{*} \rightarrow \mathbf{D}$ with monodromy in $\operatorname{PSU}(1,1)$ such that $d s^{2}=f^{*} g_{\text {st }}$. We say $f$ a developing map of the hyperbolic 
metric $d s^{2}$, which is unique up to a post-composition by a Möbius transformation in $\operatorname{PSU}(1,1)$. Moreover, the Schwarzian derivative $\{f, z\}$ of $f$ is meromorphic in $U^{*}$.

Definition 3.1. We say that $z=0$ is a cusp singularity of a hyperbolic metric $d s^{2}$ on $U^{*}$ if and only if near $z=0$ there holds $\{f, z\}=\frac{1}{2 z^{2}}+\frac{b_{0}}{z}+h(z)$, where $b_{0}$ is a constant and $h(z)$ is holomorphic near $z=0$. We say that $z=0$ is a cone singularity with angle $2 \pi \theta>0$ of $d s^{2}$ if and only if near $z=0$ there holds $\{f, z\}=\frac{1-\theta^{2}}{2 z^{2}}+\frac{c_{0}}{z}+g(z)$, where $c_{0}$ is a constant and $g(z)$ is holomorphic near $z=0$.

Lemma 3.1. The differential geometric definition of cone/cusp singularity of a hyperbolic metric in $U^{*}$ in the first paragraph of Section 2 coincides with the complex analytical one as in Definition 3.1.

We leave to the next section the proof that the complex analytical definition implies the differential geometric one. We shall prove the implication of the opposite direction in what follows.

Suppose that in the differential geometric sense, $z=0$ is a cone singularity of angle $2 \pi \theta>0$ of the hyperbolic metric $d s^{2}=e^{2 u}|d z|^{2}$ in $U^{*}=\{0<|z|<1 / 2\}$. Then, by the similar computation in [4, Lemma 3.1] and [25, Lemma, Section 3], we find that the Schwarzian derivative of $f$ equals $2\left(\frac{\partial^{2} u}{\partial z^{2}}-\left(\frac{\partial u}{\partial z}\right)^{2}\right)$, which has the form of $\frac{1-\theta^{2}}{2 z^{2}}+\frac{c_{0}}{z}+g(z)$, where $c_{0}$ is a constant, and $g(z)$ is holomorphic near $z=0$. Hence, $z=0$ is also a cone singularity in the sense of Definition 3.1. However, the same argument could not go through for $\{f, z\}$ if $z=0$ is a cusp singularity of $d s^{2}$ in the differential geometric sense. In the remaining part of this section, we shall show by a different PDE method from [25, Lemma, Section 3] that $f$ is a cusp singularity in the sense of Definition 3.1.

Suppose that $z=0$ is a cusp singularity of the hyperbolic metric $d s^{2}=e^{2 u}|d z|^{2}$ in $U^{*}=\{0<|z|<1 / 2\}$ in the differential geometric sense. Then

$$
v:=u+\ln |z|+\ln (-\ln |z|)
$$

is continuous on $U$, and we have

$$
d s^{2}=e^{2 u}|d z|^{2}=f^{*} g_{\mathrm{st}}=\frac{4\left|f^{\prime}\right|^{2}|d z|^{2}}{\left(1-|f|^{2}\right)^{2}} \quad \text { and } \quad u=\ln 2+\ln \left|f^{\prime}\right|-\ln \left(1-|f|^{2}\right) .
$$

It suffices to show that $\{f, z\}=\frac{1}{2 z^{2}}+\frac{d}{z}+\psi(z)$, where $d$ is a constant and $\psi$ is holomorphic in $U$. By computation, there holds in $U^{*}$ that

$$
\{f, z\}=2\left(\frac{\partial^{2} u}{\partial z^{2}}-\left(\frac{\partial u}{\partial z}\right)^{2}\right)=\frac{1}{2 z^{2}}+\frac{2}{z}\left(\frac{\partial v}{\partial z}\left(1+\frac{1}{\ln |z|}\right)+z \frac{\partial^{2} v}{\partial z^{2}}-z\left(\frac{\partial v}{\partial z}\right)^{2}\right)
$$

which is holomorphic in $U^{*}$ since $d s^{2}$ is hyperbolic there [25, Lemma, Section 3]. The problem is reduced to showing

Lemma 3.2. The holomorphic function

$$
F(z):=\frac{\partial v}{\partial z}\left(1+\frac{1}{\ln |z|}\right)+z \frac{\partial^{2} v}{\partial z^{2}}-z\left(\frac{\partial v}{\partial z}\right)^{2}
$$

in $U^{*}$ extends to $z=0$.

We need two lemmas for the proof of Lemma 3.2. 
Lemma 3.3. Denote $\mathbf{D}:=\{|z|<1 / 5\} \subset U$. Then $\int_{\mathbf{D}}|\nabla v|^{2}<+\infty$. Here and later on we omit in the integrals the standard Lebesgue measure $\frac{\sqrt{-1}}{2} \mathrm{~d} z \wedge \mathrm{d} \bar{z}$ on the Euclidean plane $\mathbf{C} \supset \mathbf{D}$.

Proof. The proof is divided into three steps.

Step 1. Since $g=e^{2 u}|d z|^{2}$ is a hyperbolic metric on $U^{*}$, we have $\Delta u=4 \frac{\partial^{2} u}{\partial z \partial \bar{z}}=e^{2 u}$ on $U^{*}$. Since $v=u+\ln |z|+\ln (-\ln |z|)$, we rewrite $\Delta u=e^{2 u}$ as

$$
\Delta v=h:=\frac{e^{2 v}-1}{|z|^{2}(\ln |z|)^{2}} \quad \text { in } U^{*} .
$$

Recall that $v$ is continuous in $U$ and $h$ is locally integrable in $U$. Hence, both sides of (1) can be thought of as distributions in $U$. Now we shall prove that (1) holds in $U$ in the sense of distribution.

As a distribution, the support of $\Delta v-h$ is contained in $\{z=0\}$. By [12, Theorem 2.3.4], $\Delta v-h$ equals a linear combination of the Dirac delta function $\delta_{0}$ and its partial derivatives, i.e. $\Delta v-h=\sum C_{\alpha} \partial^{\alpha} \delta_{0}$, where there are at most finitely many nonzero constants $C_{\alpha}$. Take an arbitrary multi-index $\alpha$ and fix it. We can choose a function $\phi \in C_{0}^{\infty}(U)$ such that $\partial^{\alpha} \phi(0) \neq 0$ and $\partial^{\beta} \phi(0)=0$ for all $\beta \neq \alpha$. Denote $\phi_{k}(z)=\phi(k z)$. Then

$$
h\left(\phi_{k}\right)=\int_{U} h \phi_{k} \leq \sup \left|\phi_{k}\right| \int_{\operatorname{supp} \phi_{k}} h \rightarrow 0 \quad \text { as } k \rightarrow \infty .
$$

Moreover, since $\int_{U} \Delta \phi=0$ and $v$ is continuous at $z=0$, we have

$$
\left|\Delta v\left(\phi_{k}\right)\right|=\left|\int_{U} v(z / k) \Delta \phi-\int_{U} v(0) \Delta \phi\right| \leq \int_{U}|v(z / k)-v(0)||\Delta \phi| \rightarrow 0 .
$$

Hence $(\Delta v-h)\left(\phi_{k}\right) \rightarrow 0$ as $k \rightarrow \infty$. On the other hand, $(\Delta v-h)\left(\phi_{k}\right)=C_{\alpha} k^{|\alpha|} \partial^{\alpha} \phi(0)$, which implies $C_{\alpha}=0$. Thus $\Delta v=h$ on $U$ as distributions.

Step 2. Recall that $v$ is smooth in $U^{*}$ and continuous at $z=0$. We shall prove

$$
\int_{\mathbf{D}^{*}}|\nabla v|^{2}<+\infty
$$

Choose a family $\left\{\chi_{\epsilon}: \epsilon>0\right\}$ of compactly supported non-negative smooth functions in $\mathbf{C}$ such that $\int_{\mathbf{C}} \chi_{\epsilon}=1$ and $\operatorname{supp} \chi_{\epsilon} \subset\{|z| \leq \epsilon\}$. Since $v$ is continuous on $U$, the convolutions $v_{k}:=\chi_{\frac{1}{k}} * v, k=5,6,7, \cdots$, are well defined smooth functions in $\overline{\mathbf{D}}$, which converge uniformly to $v$ on $\overline{\mathbf{D}}$ as $k \rightarrow \infty$. Moreover, since $v \in C^{\infty}\left(\mathbf{D}^{*}\right)$, as $k \rightarrow \infty,\left|\nabla v_{k}\right|^{2} \rightarrow|\nabla v|^{2}$ uniformly in any compact subsets of $\mathbf{D}^{*}$. By Fatou's lemma, we have

$$
\int_{\mathbf{D}^{*}}|\nabla v|^{2} \leq \liminf _{k} \int_{\mathbf{D}^{*}}\left|\nabla v_{k}\right|^{2}
$$

Then we show that the integrals $\int_{\mathbf{D}^{*}}\left|\nabla v_{k}\right|^{2}$ are uniformly bounded for all $k=$ $5,6,7, \cdots$. Using integration by parts, we have

$$
\int_{\mathbf{D}^{*}}\left|\nabla v_{k}\right|^{2}=\int_{\mathbf{D}}\left|\nabla v_{k}\right|^{2}=-\int_{\mathbf{D}} v_{k} \Delta v_{k}+\int_{\partial \mathbf{D}} v_{k} \frac{\partial v_{k}}{\partial \vec{n}}
$$


Recall that $v_{k} \rightarrow v$ uniformly on $\overline{\mathbf{D}}$ and $\frac{\partial v_{k}}{\partial \vec{n}} \rightarrow \frac{\partial v}{\partial \vec{n}}$ uniformly on $\partial \mathbf{D}$. The problem is reduced to showing that $\int_{\mathbf{D}}\left|\Delta v_{k}\right|$ is uniformly bounded. Actually, as $k \geq 5$, we have

$$
\begin{aligned}
\int_{\mathbf{D}}\left|\Delta v_{k}\right| & =\int_{|z|<1 / 5}\left|\int_{|\tilde{z}|<2 / 5} \chi_{1 / k}(z-\tilde{z}) \Delta v(\tilde{z})\right| \\
& \leq \int_{\mathbf{C}} \chi_{1 / k} \int_{|z|<2 / 5}|\Delta v|=\int_{|z|<2 / 5}|h|<\infty .
\end{aligned}
$$

Thus we conclude $\int_{\mathbf{D}^{*}}|\nabla v|^{2}<+\infty$.

Step 3. Denote the standard coordinate $z$ in $\mathbf{D} \subset \mathbf{C}$ by $z=x+\sqrt{-1} y$. Then $w:=\frac{\partial v}{\partial x}$ is a smooth and square integrable function on $\mathbf{D}^{*}$, which can be thought of as an square integrable function and then a distribution in $\mathbf{D}$. The partial derivative $\frac{\partial v}{\partial x}$ of the continuous function $v$ in $\mathbf{D}$ is also a distribution in $\mathbf{D}$. We shall show that the two distributions $\frac{\partial v}{\partial x}$ and $w$ coincide. Take a smooth test function $\phi$ supported in D. By the Fubini theorem and integration by parts, we have

$$
\begin{aligned}
\left(\frac{\partial v}{\partial x}-w\right)(\phi) & =\iint\left(-v \frac{\partial \phi}{\partial x}-w \phi\right)=\lim _{\epsilon \rightarrow 0^{+}} \int_{-\infty}^{\infty} d y \int_{|x|>\epsilon}\left(-v \frac{\partial \phi}{\partial x}-w \phi\right) d x \\
& =\lim _{\epsilon \rightarrow 0^{+}} \int_{-\infty}^{\infty}(v(\epsilon, y) \phi(\epsilon, y)-v(-\epsilon, y) \phi(-\epsilon, y)) d y=0 .
\end{aligned}
$$

The similar statement holds for $\frac{\partial v}{\partial y}$. Therefore, we complete the proof.

Lemma 3.4. $F(z)$ is in $L^{2-\epsilon}(\mathbf{D})$ for all $0<\epsilon<1$. In particular, $z=0$ is at most a simple pole of $F(z)$.

Proof. By Lemma 2.1, both $\frac{\partial v}{\partial z}$ and $\frac{\partial v}{\partial \bar{z}}$ belong to $L^{2}(\mathbf{D})$. Then the first summand $\frac{\partial v}{\partial z}\left(1+\frac{1}{\ln |z|}\right)$ in $F(z)$ also lies in $L^{2}(\mathbf{D})$. Defining $\tilde{v}:=z v$, we have

$$
\Delta \tilde{v}=4 \frac{\partial v}{\partial \bar{z}}+z \Delta v
$$

Since both $\frac{\partial v}{\partial \bar{z}}$ and $z \Delta v=\frac{e^{2 v}-1}{|z|(\ln |z|)^{2}}$ belong to $L^{2}(\mathbf{D})$, we have $\Delta \tilde{v} \in L^{2}(\mathbf{D})$ and then $\tilde{v} \in W^{2,2}(\mathbf{D})$. By the Sobolev embedding theorem, we obtain $\frac{\partial \tilde{v}}{\partial z}=v+z \frac{\partial v}{\partial z} \in L^{p}(\mathbf{D})$ for any $p>1$. Since $v \in L^{p}(\mathbf{D})$ as well, we have $z \frac{\partial v}{\partial z} \in L^{p}(\mathbf{D})$ for any $p>1$. We now claim that the third summand $z\left(\frac{\partial v}{\partial z}\right)^{2}$ in $F(z)$ belongs to $L^{2-\epsilon}(\mathbf{D})$ for all $0<\epsilon<1$. In fact, defining $\frac{1}{p}:=\frac{\epsilon}{2}$ and $\frac{1}{q}:=\frac{2-\epsilon}{2}$, by the Hölder inequality, we obtain

$$
\int_{\mathbf{D}}\left|z\left(\frac{\partial v}{\partial z}\right)^{2}\right|^{2-\epsilon} \leq\left(\int_{\mathbf{D}}\left|z \frac{\partial v}{\partial z}\right|^{(2-\epsilon) p}\right)^{1 / p}\left(\int_{\mathbf{D}}\left|\frac{\partial v}{\partial z}\right|^{(2-\epsilon) q}\right)^{1 / q}<\infty
$$

As long as the the second summand $z \frac{\partial^{2} v}{\partial z^{2}}$ in $F(z)$ is concerned, since $\frac{\partial^{2} \tilde{v}}{\partial z^{2}}=2 \frac{\partial v}{\partial z}+z \frac{\partial^{2} v}{\partial z^{2}} \in$ $L^{2}(\mathbf{D})$, we have $z \frac{\partial^{2} v}{\partial z^{2}} \in L^{2}(\mathbf{D})$. Therefore, we have proved $F(z) \in L^{2-\epsilon}(\mathbf{D})$. Since $F$ is holomorphic and integrable in $\mathbf{D}^{*}, z=0$ is at most a simple pole of $F$.

Proof of Lemma 3.2. We prove by contradiction. Suppose that $F(z)$ has a simple pole at $z=0$ with residue $-\lambda^{2} / 4$, where $\lambda \in \mathbf{C}^{*}$ and $\Re \lambda \geq 0$. Take a developing map $f: U^{*} \rightarrow \mathbf{D}$ of the restriction of the hyperbolic metric $d s^{2}$ to $U^{*}$. Moreover, we have $\{f, z\}=\frac{1}{2 z^{2}}-\frac{2 F(z)}{z}=\frac{1-\lambda^{2}}{2 z^{2}}+\frac{d}{z}+\psi$ for some constant $d$ and a holomorphic function $\psi$ in $U$. In each small disc lying in $U^{*}:=U \backslash\{0\}, f$ is the ratio of two linear 
independent solutions of the following Fuchsian equation $\frac{d^{2} y}{d z^{2}}+\frac{1}{2}\left(\frac{1-\lambda^{2}}{2 z^{2}}+\frac{d}{z}+\psi\right) y=0$, whose two indicial exponents are $(1+\lambda) / 2$ and $(1-\lambda) / 2$ with difference $\lambda$.

If $\lambda \notin \mathbf{Z}$, it follows from the Frobenius method [27, p.39] that there exists a small neighborhood, say $V$, of $z=0$, and another complex coordinate $\xi$ of $V$ centered at $z=0$ such that $f$ has the form of $\xi^{\lambda}$ in each small disk of $V^{*}:=V \backslash\{0\}$, where the details of computation is the same with [8]. Since $f$ takes values in $\mathbf{D}^{*}, \lambda$ must be positive and $z=0$ is a cone singularity of $d s^{2}$ with angle $2 \pi \lambda$. Contradiction!

If $\lambda \in \mathbf{Z}_{\neq 0}$, then by a combination of the Frobenius method and the fact that $f$ takes values in $\mathbf{D}^{*}$ and has monodromy in $\operatorname{PSU}(1,1)$, we find that $\lambda$ is a positive integer and $f$ has form $\xi^{\lambda}$ in another complex coordinate chart $(V, \xi)$ centered at $z=0$. It implies that $z=0$ is also a cone singularity of $d s^{2}$ of angle $2 \pi \lambda$. This is a contradiction.

\section{At last we prove}

The necessary part of Theorem 2.2. By the similar argument as [4, Lemma 2.1], there exists a projective function $f: \mathfrak{X} \backslash \operatorname{supp} \mathfrak{D} \rightarrow \mathbf{D}$ with monodromy in $\operatorname{PSU}(1,1)$ such that $d s^{2}=f^{*} g_{\mathrm{st}}$. We say $f$ a developing map of the hyperbolic metric $d s^{2}$, which is unique up to a post-composition of a Möbius transformation in $\operatorname{PSU}(1,1)$. It follows from the proven part of Lemma 3.1 that $f$ is compatible with $\mathfrak{D}$.

\section{Sufficient part of Theorem 1.2, an example and three questions}

To complete the proof of Lemma 3.1, we need only to show that Definition 3.1 for cone and cusp singularities of a hyperbolic metric $d s^{2}$ in $U^{*}=\{0<|z|<1 / 2\}$ implies the differential geometric definition of them. Actually, the argument is similar as in the proof of Lemma 2.2. If $\theta \notin \mathbf{Z}$, then by only using the Frobenius method, we find easily that $z=0$ is a cone singularity of angle $2 \pi \theta$ as $\theta>0$, and it is a cusp singularity as $\theta=0$. If $\theta$ is an integer greater than one, since $|f|<1$, we could rule out the possibility that $f$ may have the logarithmic singularity at $z=0$ and find that $z=0$ is a cone singularity of angle $2 \pi \theta_{j}$.

Then we prove

The sufficient part of Theorem 2.2. Suppose that $f: \mathfrak{X} \backslash \operatorname{supp} \mathfrak{D} \rightarrow \mathbf{D}$ is a projective function which is compatible with $\mathfrak{D}$ and has the monodromy in $\operatorname{PSU}(1,1)$. Then $f^{*} g_{\text {st }}$ is a hyperbolic metric on $\mathfrak{X} \backslash \operatorname{supp} \mathfrak{D}$. It follows from Lemma 3.1 that the metric $f^{*} g_{\text {st }}$ represents $\mathfrak{D}$.

Before giving the details of Example 2.1, we need an equivalent version of Theorem 2.2 as follows.

There exists a hyperbolic metric $d s^{2}$ representing an $\mathbf{R}$-divisor $\mathfrak{D}=\sum_{j}\left(\theta_{j}-1\right) \mathfrak{P}_{j}$ with $\theta_{j} \geq 0$ on a Riemann surface $\mathfrak{X}$ if and only if there exists a projective function $f: \mathfrak{X} \backslash \operatorname{supp} \mathfrak{D} \rightarrow \mathbf{H}:=\{w \in \mathbf{C}: \Im w>0\}$ such that $f$ is compatible with $\mathfrak{D}$ and the monodromy of $f$ lies in the holomorphic automorphism group

$$
\operatorname{PSL}(2, \mathbf{R})=\left\{w \longmapsto \frac{a w+b}{c w+d}: a, b, c, d \in \mathbf{R}, a d-b c=1\right\}
$$

of $\mathbf{H}$. Moreover, $d s^{2}$ coincides with the pullback $f^{*} \widetilde{g_{\mathrm{st}}}$ of the standard hyperbolic metric $\widetilde{g_{\mathrm{st}}}:=\frac{4|d w|^{2}}{(\Im w)^{2}}$ on $\mathbf{H}$ by $f$. We say $f$ a developing map of the metric $d s^{2}$, which is uniquely determined up to a post-composition with an automorphism of $\mathbf{H}$. 
Denote $\omega:=-\sqrt{-1} h(z) d z=\sum_{j=1}^{\infty}\left(\frac{-\sqrt{-1} a_{j}}{z-z_{j}}\right) d z$. Since $\sum_{n=1}^{\infty} a_{n}$ is a convergent series of positive numbers, we observe that the multi-valued function $\int_{0}^{z} \omega$ on $\mathbf{D} \backslash\left\{z_{j}\right\}_{j=1}^{\infty}$ has monodromy in $\{w \mapsto w+t: t \in \mathbf{R}\} \subset \operatorname{PSL}(2, \mathbf{R})$ such that its imaginary part $\Im\left(\int_{0}^{z} \omega\right)$ is single-valued and has a lower bound. Hence, there exists a real number $\lambda_{0}$ such that for all $z \in \mathbf{D} \backslash\left\{z_{j}\right\}_{j=1}^{\infty}$

$$
\lambda_{0}+\Im\left(\int_{0}^{z} \omega\right) \geq 0 .
$$

Hence

$$
f_{\lambda}(z)=\sqrt{-1} \lambda+\int_{0}^{z} \omega, \quad \lambda \in\left(\lambda_{0}, \infty\right)
$$

is a family of projective functions on $\mathbf{D} \backslash\left\{z_{j}\right\}_{j=1}^{\infty}$ taking values in $\mathbf{H}$ and having monodromy in $\{w \mapsto w+t: t \in \mathbf{R}\} \subset \operatorname{PSL}(2, \mathbf{R})$. We claim that $d s_{\lambda}^{2}:=f_{\lambda}^{*}\left(\widetilde{g_{\mathrm{st}}}\right)$ is a family of hyperbolic metrics representing the divisor $\mathfrak{D}=(h)$, i.e. $d s_{\lambda}^{2}$ has cusp singularities at simple poles of $h$, and has a cone singularity at each zero $w$ of $h$ with cone angle $2 \pi\left(1+\operatorname{ord}_{w}(h)\right)$. Actually, $f_{\lambda}(z)$ equals $\left(-\sqrt{-1} a_{j}\right) \log \left(z-z_{j}\right)$ plus a a multi-valued holomorphic function near $z_{j}$, so $\{f, z\}=\frac{1}{\left(z-z_{j}\right)^{2}}+\cdots$ there. Hence $z_{j}$ is a cusp singularity of $d s_{\lambda}^{2}$. Near each zero $w$ of $h(z)$ with multiplicity $\ell$, we have $\frac{d}{d z}\left(f_{\lambda}(z)\right)=(-\sqrt{-1}) h(z)=(z-w)^{\ell} g(z)$, where $g(z)$ is holomorphic at $w$ and $g(w) \neq 0$. Hence near $w,\{f, z\}=\frac{1-(\ell+1)^{2}}{(z-w)^{2}}+\cdots$, which implies that $w$ is a cone singularity of $d s_{\lambda}^{2}$ with angle $2 \pi(1+\ell)$.

By now, we have proved the statements in the first paragraph of Example 2.1. For the second one, we need the following elementary lemma.

Lemma 4.1. Let $a_{1}, \cdots, a_{N}$ be $N \geq 2$ positive numbers and $z_{1}, \cdots, z_{N}$ be distinct complex numbers in the disc $\{|z|<R\}$, where $R$ is a positive constant. Then, the rational function $\sum_{j=1}^{N} \frac{a_{j}}{z-z_{j}}$ has $(N-1)$ zeros (counting multiplicities) on the disc $\{|z|<R\}$. The meromorphic function $h$ on $\mathbf{C} \backslash\{|z|=1\}$ in Example 2.1 has no zero in $\{z \in \mathbf{C}:|z| \geq 1\}$.

Proof. Taking a complex number $\xi$ such that $\Im \xi \leq-R$, we find

$$
\Im\left(\xi-z_{j}\right)<0 \text { and } \Im \frac{a_{j}}{\xi-z_{j}}>0,
$$

which implies $\sum_{j=1}^{N} \frac{a_{j}}{\xi-z_{j}} \neq 0$. Observing that $\Im z=-R$ is a tangent line to the circle $\{|z|=R\}$, we could prove the first statement by arguing on each half plane defined by each tangent line to the circle and disjoint from the disc $\{|z|<R\}$. The second one follows from the similar argument. We also note that $h$ extends holomorphically to each point on the circle $\partial \mathbf{D}=\{|z|=1\}$, which is not a limit point of $\left\{z_{j}\right\}$.

Then we prove that $h_{0}(z)=: \sum_{j=1}^{\infty} \frac{a_{j}}{z-z_{j}}$ has the same number of zeros as $f_{N}(z):=$ $\sum_{\left|z_{j}\right| \leq r_{N}} \frac{a_{j}}{z-z_{j}}$ on the disc $\left\{|z|<r_{N}:=1-\frac{1}{2 N}\right\}$ when $N$ is sufficiently large, where $a_{j}=\frac{1}{2 j^{3}(2 j+1)}$ and $z_{j}=1-\frac{1}{2 j-1}$. At first we show that on the circle $\left\{|z|=r_{N}\right\}$ there holds $\left|f_{N}(z)\right|>\left|g_{N}(z)\right|$ when $N$ is sufficiently large, where

$$
g_{N}(z):=h_{0}(z)-f_{N}(z)=\sum_{\left|z_{j}\right|>r_{N}} \frac{a_{j}}{z-z_{j}} .
$$


In fact, on the circle $\left\{|z|=r_{N}\right\}$, we have

$$
\left|g_{N}(z)\right| \leq \sum_{\left|z_{j}\right|>r_{N}} \frac{a_{j}}{\left|z_{j}\right|-r_{N}} \leq 2 N(2 N+1) \sum_{\left|z_{j}\right|>r_{N}} a_{j} \leq \sum_{j>N} \frac{1}{j^{2}}
$$

Moreover, denoting by $z=i r_{N} e^{i \theta}$ a point $z$ on this circle, by computation, we have

$$
f_{N}(z)=e^{-i \theta} \sum_{\left|z_{j}\right|<r_{N}} \frac{a_{j}}{i r_{N}-z_{j} e^{-i \theta}}=e^{-i \theta} \sum_{\left|z_{j}\right|<r_{N}} \frac{a_{j}\left(-i r_{N}-z_{j} e^{i \theta}\right)}{\left|i r_{N}-z_{j} e^{-i \theta}\right|^{2}},
$$

and recalling $z_{1}=0$, we obtain

$$
\left|f_{N}(z)\right|=\left|e^{i \theta} f_{N}(z)\right| \geq\left|\Im\left(e^{i \theta} f_{N}(z)\right)\right|=\sum_{\left|z_{j}\right|<r_{N}} \frac{a_{j}\left(r_{N}+z_{j} \sin \theta\right)}{\left|i r_{N}-z_{j} e^{-i \theta}\right|^{2}} \geq \frac{a_{1}}{r_{N}} \geq a_{1}
$$

and prove the inequality $\left|f_{N}(z)\right|>\left|g_{N}(z)\right|$ on the circle. Since both $h_{0}$ and $f_{N}$ have $N$ simple poles on the disc $\left\{|z|<r_{N}\right\}$ and $\left|h_{0}(z)-g_{N}(z)\right|=\left|f_{N}(z)\right|>\left|-g_{N}(z)\right|$ on the circle $\left\{|z|=r_{N}\right\}$, by the Rouché theorem, $h_{0}(z)$ has the same number of zeros as $f_{N}(z)$ on the disc $\left\{|z|<r_{N}\right\}$. By Lemma 4.1, counting multiplicities, we find that $f_{N}(z)$ has $(N-1)$ zeros on the disc.

Restricting $h_{0}(z)$ to the interval $\left(1-\frac{1}{2 j-1}, 1-\frac{1}{2 j+1}\right)$, we find by the intermediate value theorem that $h_{0}(z)$ has a zero $w_{j}$ lying on this interval. Since $h_{0}(z)$ has at most $(N-1)$ zeros (counting multiplicities) on the interval $\left(0,1-\frac{1}{2 N}\right)$ by the result in the above paragraph, all the zeros of $h_{0}(z)$ lie on the interval $(0,1)$ and $w_{j}$ is the unique zero of $h_{0}(z)$ lying in $\left(1-\frac{1}{2 j-1}, 1-\frac{1}{2 j+1}\right)$. By now we have completed the explanation of Example 2.1.

At last, we propose the following two questions.

Question 4.1. Use the notions in Theorem 1.1 and assume that $\theta_{j}$ 's are nonnegative rational numbers. What is the necessary and sufficient condition for $D=$ $\sum_{j=1}^{n}\left(\theta_{j}-1\right) P_{j}$ under which the monodromy group of the developing map $f$ of the hyperbolic metric $d s^{2}$ representing $D$ on a compact Riemann surface $X$ is discrete in $\operatorname{PSU}(1,1)$ ? It is the case when $\theta_{j} \in\{0,1 / 2,1 / 3, \cdots\}$ by the Uniformization Theory. Also a conceptual necessary and sufficient condition was given in [2, Theorem 3.29] for the case of 3 singularities on the Riemann sphere, which has yet to be expressed in terms of $\theta_{1}, \theta_{2}$ and $\theta_{3}$.

Question 4.2. It is interesting for us to investigate the existence and the uniqueness of hyperbolic metrics with isolated singularities on noncompact Riemann surfaces, which seems to be an open problem to the best of our knowledge. A major problem [17] in the theory of Bergman spaces, which has been open for 50 years or so, forms a special case of the existence problem of singular hyperbolic metrics. Precisely speaking, the problem mentioned in [17] asks for a characterization of the critical set of a holomorphic self-map of the unit disc. On the other hand, it follows from Theorem 2.2 that such a self-map is equivalent to a hyperbolic metric with cone singularities of cone angles of $2 \pi$ times positive integers on the unit disc.

Question 4.3. Given a convergent series $\sum_{j=1}^{\infty} a_{n}$ of positive numbers and a sequence of positive integers $m_{1}, m_{2}, \cdots$, does there exist a closed discrete subset $\left\{z_{j}\right\}$ of $\mathbf{D}$ such that $h(z)=\sum_{j=1}^{\infty} \frac{a_{j}}{z-z_{j}}$ have infinitely many zeros $w_{1}, w_{2}, \cdots$ with 
multiplicities $m_{1}, m_{2}, \cdots$, respectively? This question is motivated by the relationship between the multiplicities of zeros of $h(z)$ and the cone angles of the hyperbolic metrics $d s_{\lambda}^{2}$ in Example 2.1, and its answer is positive if $1=m_{1}=m_{2}=\cdots$.

Acknowledgement. The authors would like to express their great gratitude to Professor Alexandre Eremenko, Mr Yu Feng and Professor Dangzheng Liu for several valuable conversations about Example 2.1. They also would like to thank the two respectable anonymous referees for their careful reading and valuable comments which improved the manuscript greatly. The first author is supported in part by China Scholarship Council, the second author by ERC ALKAGE and the last author by the National Natural Science Foundation of China (grant nos. 11571330 and 11971450) and the Fundamental Research Funds for the Central Universities.

\section{References}

[1] Anderson, G. D., T. Sugawa, M. K. Vamanamurthy, and M. Vuorinen: Twicepunctured hyperbolic sphere with a conical singularity and generalized elliptic integral. - Math. Z. 266:1, 2010, 181-191.

[2] Beukers, F.: Gauss' hypergeometric function. - In: Arithmetic and geometry around hypergeometric functions, Progr. Math. 260, Birkhäuser, Basel, 2007, 23-42.

[3] BorceA, J.: Equilibrium points of logarithmic potentials induced by positive charge distributions I: generalised de Bruijn-Springer relations. - Trans. Amer. Math. Soc. 359, 2007, 3209-3237.

[4] Chen, Q., W. Wang, Y. Wu, and B. Xu: Conformal metrics with constant curvature one and finitely may conical singularities on compact Riemann surfaces. - Pacific J. Math. 273:1, 2015, 75-100.

[5] Clunie, J., A. Eremenko, and J. Rossi: On equilibrium points of logarithmic and Newtonian potentials. - J. London Math. Soc. 47:2, 1993, 309-320.

[6] Eremenko, A., J. K. LAngley, and J. Rossi: On the zeros of meromorphic functions of the form $\sum_{k=1}^{\infty} \frac{a_{k}}{z-z_{k}}$. - J. Anal. Math. 62, 1994, 271-286.

[7] Farkas, H. M., and I. Kra: Riemann surfaces. Second edition. - Grad. Texts in Math. 71, Springer-Verlag, New York, 1992.

[8] Feng, Y., Y. Shi, and B. Xu: Isolated singularities of conformal hyperbolic metrics. - Chinese J. Contemp. Math. 40:1, 2019, 15-26.

[9] Feng, Y., Y. ShI, and B. Xu: Characterizing isolated singularities of conformal hyperbolic metrics. - arXiv 1906.07522

[10] Feng, Y., Y. Shi, J. Song, and B. Xu: Subharmonic functions, meromorphic differentials and hyperbolic metrics with isolated singularities on Riemann surfaces. - In preparation.

[11] Heins, M.: On a class of conformal metrics. - Nagoya Math. J. 21, 1962, 1-60.

[12] Hörmander, L.: The analysis of linear partial differential operators I. Second edition. Springer Study Edition, Springer-Verlag, Berlin, 1990.

[13] Langley, J. K.: Equilibrium points of logarithmic potentials on convex domains. - Proc. Amer. Math. Soc. 135:9, 2007, 2821-2826.

[14] Langley, J. K., and J. Rossi: Meromorphic functions of the form $f(z)=\sum_{n=1}^{\infty} a_{n} /\left(z-z_{n}\right)$. - Rev Mat. Iberoamericana 20, 2004, 285-314.

[15] Langley, J. K., and J. Rossi: Critical points of certain discrete potentials. - Complex Variables 49, 2004, 621-637.

[16] LuO, F.: Monodromy groups of projective structures on punctured surfaces. - Invent. Math. $111,1992,541-555$. 
[17] Kraus, D.: Critical sets of bounded analytic functions, zero sets of Bergman spaces and nonpositive curvature. - Proc. Lond. Math. Soc. 106:3, 2013, 931-956.

[18] Kraus, D., and O. Roth: The behaviour of solutions of the Gaussian curvature equation near an isolated boundary point. - Math. Proc. Cambridge Philos. Soc. 145:3, 2008, 643-667.

[19] Kraus, D., O. Roth, and T. Sugawa: Metrics with conical singularities on the sphere and sharp extensions of the theorems of Landau and Schottky. - Math. Z. 267:3-4, 2011, 851-868.

[20] McOwen, R.: Point singularities and conformal metrics on Riemann surfaces. - Proc. Amer. Math. Soc. 103, 1988, 222-224.

[21] Nitsche, J.: Über die isolierten Singularitäten der Lösungen von $\Delta u=e^{u}$. - Math. Z. 68, $1957,316-324$.

[22] Picard, É.: De l'intégration de l'équation $\Delta u=e^{u}$ sur une surface Riemann fermée. - J. Reine Angew. Math. 130, 1905, 243-258.

[23] Song, J., Y. Cheng, B. Li, and B. Xu: Drawing cone spherical metrics via Strebel differentials. - Int. Math. Res. Not. IMRN, rny103, https://doi.org/10.1093/imrn/rny103.

[24] Thurston, W. P.: Three-dimensional geometry and topology. Volume 1. - Princeton Math. Ser. 35, Princeton Univ. Press, Princeton, NJ, 1997.

[25] Troyanov, M.: Metrics of constant curvature on a sphere with two conical singularities. Lecture Notes in Math. 1410, Springer, NY, 1989, 296-308.

[26] Troyanov, M.: Prescribing curvature on compact surfaces with conical singularities. - Trans. Amer. Math. Soc. 324, 1991, 793-821.

[27] Yoshida, M.: Fuchsian differential equations: with special emphasis on the Gauss-Schwarz theory. - Aspects Math. 11, Vieweg, Braunschweig, 1987.

[28] ZHANG, T.: Asymptotic properties of the hyperbolic metric on the sphere with three conical singularities. - Bull. Korean Math. Soc. 51:3, 2014, 1485-1502.

Received 11 January 2019 • Received 28 June $2019 \bullet$ Accepted 9 September 2019 